\title{
Factors moderating blocking in human place learning: The role of task instructions
}

\author{
Oliver Hardt \\ McGill University, Montreal, Quebec, Canada \\ AND \\ Almut Hupbach and Lynn Nadel \\ University of Arizona, Tucson, Arizona
}

\begin{abstract}
Cognitive map theory assumes that novel environmental information is automatically incorporated into existing cognitive maps as a function of exploration. Reports of blocking in place learning cast doubt on this claim. In these studies, subjects were first trained to find a place, using a set of landmarks (Set A). Then novel landmarks (Set B) were added for additional trials. Subsequent removal of the Set A landmarks showed that the novel landmarks alone were insufficient for successful navigation. We investigated whether instructing human subjects to explore the environment can moderate blocking. First, we demonstrated that blocking is absent in a computer implementation of the Morris water maze (MWM) in which subjects are instructed to explore. We then studied why others found blocking in a different MWM implementation, in which the task instructions did not suggest exploration. In experiments that faithfully replicated this MWM variant, we found that subjects did not acquire cognitive maps and that blocking was attenuated when instructions were provided that encouraged exploration. Together, these findings indicate that blocking in human place learning may reflect a performance deficit, not a learning deficit, and that instructions can moderate blocking. Our results thus support the automatic update assumption of cognitive map theory.
\end{abstract}

Cognitive map theory (O’Keefe \& Nadel, 1978) proposes that allocentric representations are dynamically updated after a mismatch between the actual environment and its internal representation has been detected and explored. Furthermore, this updating is assumed to occur whether or not there have been changes in the reward characteristics of the environment. These assertions suggest that certain basic associative learning phenomena - for example, blocking and overshadowing - might not be observed during the acquisition and use of cognitive maps: Provided that newly added spatial information is noticed and explored, cognitive map theory predicts that the organism's allocentric representation of the environment will be modified accordingly. Since spatial behavior based on egocentric strategies and representations is proposed to rely on brain systems and learning mechanisms that are different from those relied on by cognitive-map-based spatial behavior, newly added spatial information may not be acquired in this case unless there are changes in the reward characteristics of the environment. Thus, cognitive map theory assumes that the rules of associative learning govern the acquisition of egocentric spatial representations but do not directly predict updating of allocentric representations as a function of exploration alone.
The blocking phenomenon (Kamin, 1969) is ideally suited for studying the memory-updating prediction of cognitive map theory. In a blocking experiment, subjects are first exposed to a contingency (e.g., $A \rightarrow B$ ), learning that $A$ predicts $B$. After acquisition, another stimulus (C) is added, such that $(A+C) \rightarrow B$ (compound training). Kamin originally reported that subjects fail to acquire a conditioned response to $C$ because, as he and others (e.g., Rescorla \& Wagner, 1972) argued, $B$ was already fully predicted by $A$. The assertion of cognitive map theory that maps can be automatically updated leads to a contrasting prediction - namely, that subjects will add stimulus $C$ to their allocentric representation of a test environment during compound training, provided $C$ has been noticed and explored, even though this learning need not increase the predictability of places. Thus, cognitive map theory expects that blocking will not be observed in the acquisition of cognitive maps (Morris, 1981; Nadel \& Willner, 1980). Associative learning accounts do not directly predict updating of allocentric representations as a function of exploration, although some associative theories are capable of modeling post hoc, and thereby explaining, cases of such learning in which blocking effects are not observed (e.g., Mackintosh, 1975; Miller \& Shettleworth, 2007; Wagner, 1981; Wagner \& Brandon, 1989). It therefore

o. Hardt, oliver.hardt@mac.com 
depends on the specific class of theories of associative learning whether they predict a priori or account post hoc for blocking in allocentric learning.

This question has been addressed in a number of animal studies (e.g., Biegler \& Morris, 1999; Brown, Yang, \& DiGian, 2002; Chamizo, Sterio, \& Mackintosh, 1985; Hayward, McGregor, Good, \& Pearce, 2003; McGregor, Hayward, Pearce, \& Good, 2004; Pearce, Ward-Robinson, Good, Fussell, \& Aydin, 2001; Rodrigo, Chamizo, McLaren, \& Mackintosh, 1997; Sanchez-Moreno, Rodrigo, Chamizo, \& Mackintosh, 1999), but the results so far have been inconclusive, since in some studies animals updated their spatial representations, whereas in others blocking effects were found instead. Several studies have addressed the question of whether blocking (and overshadowing) effects are affected by the type of spatial stimuli used in the environment, such as geometry and proximal cues (e.g., Doeller \& Burgess, 2008; Graham, Good, McGregor, \& Pearce, 2006; Gray, Bloomfield, Ferrey, Spetch, \& Sturdy, 2005; Hayward, Good, \& Pearce, 2004; Pearce, Good, Jones, \& McGregor, 2004; Wall, Botly, Black, \& Shettleworth, 2004). Again, the results are mixed, and a discussion of this complex literature is beyond our present scope.

The development of a computer-generated simulation of the Morris water maze (MWM) by Jacobs, Laurance, and Thomas (1997) made it possible to address this issue in humans. Behavior in this task seems to reflect the generation and use of a cognitive map of the environment. First, Jacobs et al. (1997) showed that a proximal cue did not overshadow acquisition of simultaneously available distal cues. Removal of the proximal cue did not impair spatial performance, which was indistinguishable from performance when there was training with distal cues alone. Second, Jacobs, Thomas, Laurance, and Nadel (1998) demonstrated that although the ability to find the hidden platform was disrupted by the removal of all the distal cues, it did not depend on individual distal cues, because removal of any arbitrary subset of cues failed to impair task performance. These results indicate that spatial learning in this computer implementation leads to the generation of cognitive maps that encompass most, if not all, distal cues of the environment (cf. O'Keefe \& Nadel, 1978).

Hamilton and Sutherland (1999) used an MWM implementation that was similar in some, but not all, respects to the version developed by Jacobs et al. (1997). In a standard blocking design, subjects were trained in two phases to locate a hidden platform in this MWM variant. In the first phase, four distal cues $(A, B, C$, and $D)$ were presented on the walls of the room. During subsequent compound training, four more distal cues $(E, F, G$, and $H)$ were added. Then the ability to accurately search for the target in the correct area was tested during a probe trial with the target absent and the four original cues $(A-D)$ removed. The blocking group participated in both training phases, whereas the control group was exposed only to compound training. The subjects in the blocking group were not able to accurately search for the target in the probe trial solely on the basis of the cues that were introduced during com- pound training $(E-H)$, whereas the subjects in the control group were able to do so.

This blocking effect and the results of Jacobs et al. (1997) seemed to be at odds with each other, and the question arose as to why Hamilton and Sutherland's (1999) subjects did not incorporate the additional spatial information into their existing representations. We therefore first considered whether the blocking effect was contingent on some parameters of the water maze variant used by Hamilton and Sutherland by asking whether blocking would also be observed in the MWM implementation developed by Jacobs and colleagues. We found that blocking was absent in the latter. We then explored what kind of spatial representations the subjects acquired in the MWM implementation in which Hamilton and Sutherland found a blocking effect. Specifically, we tried to determine whether the subjects created a cognitive map-like representation of the entire environment or whether some cues were more likely to be incorporated and used than others. To this end, we replicated the Hamilton and Sutherland version of the water maze task and removed half of the cues after training. Our results showed that the subjects preferentially encoded a small subset of the available cues, unlike in the implementation of Jacobs et al. (1998), in which performance did not depend on the presence of specific distal cues.

We then asked what factor might moderate the difference in learning behavior and the spatial representations formed in these two water maze environments. Taking a closer look at the respective tasks, we found dissimilarities in the experimental protocols (e.g., number of training trials, task instructions, dimensions of the environment, nature of the distal cues) and the specifics of the software implementations (e.g., field of view, speed of movement, optical flow, quality of the rendered scene). A potentially critical difference between the two studies was that when blocking was absent, subjects had been explicitly instructed to explore the environment. Such instructions had not been provided for the MWM task in which blocking was detected, since it had been designed to replicate the animal version as precisely as possible, in which instructions cannot be given. As was pointed out above, cognitive map theory stresses that maps are updated as a function of exploratory behavior. We thus tested whether instructions to explore would influence the occurrence of blocking in the MWM implementation in which it had been previously found. Our results showed an attenuation of the blocking effect, suggesting that these instructions increased the likelihood that changes in the environment would be noticed and incorporated into existing spatial representations. However, our results also suggested that other factors were likely involved in the expression of this knowledge, so that under some conditions additional spatial information was not accessed, although it was available - that is, had been acquired.

\section{EXPERIMENT 1}

The first experiment tested whether blocking occurs in human place learning in a computer-generated MWM 
(cMWM) when an experimental protocol is used in which it has been shown that subjects create cognitive maps of the environment (Jacobs et al., 1997; Jacobs et al., 1998).

\section{Method}

\section{Subjects}

Sixty-four University of Arizona undergraduate students (18-24 years old, $M=20.4, S D=1.3 ; N_{\text {male }}=32=50 \%$ ) served as subjects. They received course credit for participation. The Behavioral Science Committee at the University of Arizona and the Psychology Department's Internal Review Board approved all the experiments reported here.

\section{Apparatus}

A personal computer (Pentium III, Windows 95) ran the CGArena software (Jacobs et al., 1997), displaying the cMWM on a standard CRT desktop monitor. All software parameters (i.e., dimensions, textures, timers, rendering, etc.) were set as described by Jacobs et al. (1997; Jacobs et al., 1998).

The environment consisted of a circular arena that was placed in the center of a larger square room. The room's ceiling was gray. A red brick stone pattern was displayed on the circular arena wall (see Figure 1A). The north wall's distal cues were a door and two windows, displayed against a featureless gray background. A Roman aqueduct with five arches was displayed on the east wall. The distal cues of the south wall were three windows on a gray background, and the west wall's cues consisted of a relatively large window, centered in the middle of the wall, which itself was covered with a regular pattern of small light-gray squares. In some experimental conditions, the walls were devoid of distal cues for some time and then were colored in a featureless gray. The target was a pink square located in the center of the arena's northwest quadrant. The target became visible only when subjects "stepped" onto it.

The cMWM was displayed from the perspective of an averagesized person standing on the cMWM floor. When the subject stood right in front of the arena wall and faced it, the upper half of the computer monitor displayed a part of the room, and the lower half the arena wall. When the subject turned around at this position, a large portion of the room (walls, ceiling, floor) became visible.

Movement was controlled by the four arrow keys of the keyboard: The $\uparrow$ key moved the subject forward, the $\downarrow$ key moved the subject backward, and the $\leftarrow$ and the $\rightarrow$ keys rotated the subject's heading without accompanying forward or backward locomotion. Pressing a rotation key and the forward or backward key combined forward or backward locomotion with a simultaneous change in heading.

\section{Procedure}

The subjects were randomly assigned to an experimental condition (see Table 1). The subjects were told how to navigate the computer-generated space with the arrow keys and were told that their task was to repeatedly find a hidden target, which would always be in the same position. The subjects were encouraged to explore the room by turning around in place, after finding the target. No information was provided at any time about the number of trials, trial duration, and the absence of the target during the first probe trial, and the subjects were not told that the distal cues would change at some point. The instructions were the same as those used by Jacobs et al. (1997; Jacobs et al., 1998).

A practice trial preceded acquisition, which allowed the subjects to learn how to navigate the cMWM. The practice environment was identical in layout and dimension to the cMWM but otherwise was clearly dissimilar. There was no target, and the room's walls were painted in bright featureless colors (red, blue, yellow, and green), with no distal cues. The ceiling was solid white, the floor solid gray, and the arena wall solid purple. The subjects self-terminated practice by pressing the space bar, which started the first acquisition trial.

Acquisition. Acquisition consisted of two phases, with eight trials each. In the first phase, only one set of distal cues was present. During the second phase, compound training, another set of distal cues was added. The blocking group participated in both phases, the control group only in compound training (see Table 1).

Eight different starting positions were used (see Figure 1A), once per phase. A sequence of starting positions was pseudorandomly determined and administered to all the subjects. The subjects started each trial facing the wall of the arena. If they did not find the target within $180 \mathrm{sec}$, the trial ended automatically. Stepping on the target made it immediately visible. Simultaneously, a clicking sound was played over the headphones. When on the target, the subjects were unable to move off it, but pressing the space bar within $30 \mathrm{sec}$ terminated the trial, after which it ended automatically.

The subjects automatically returned to the practice room after each acquisition trial. From there, the next trial was initiated by pressing the space bar. The intertrial interval was therefore variable.

Probe trials. In order to assess knowledge of the target's location, it was removed in the two probe trials that followed the last acquisition trial. During both trials, only one set of distal cues was displayed. For both groups, these were the cues that had been added
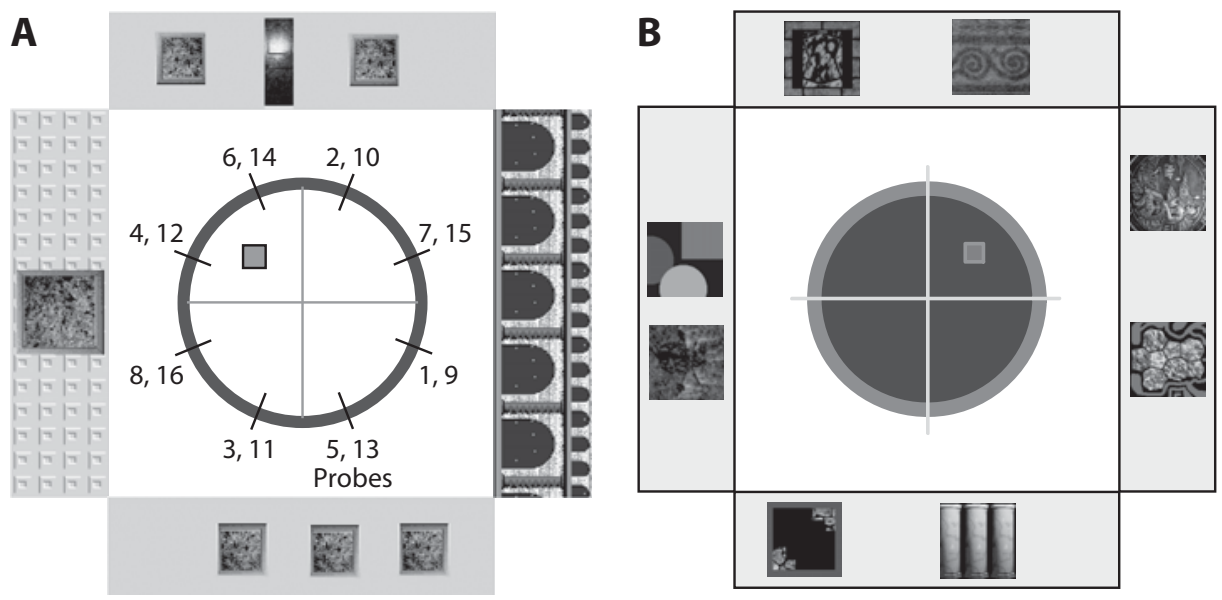

Figure 1. Bird's-eye, schematic views of the computer-generated Morris water mazes (cMWMs) used in the experiments. The target is the square located in one arena quadrant. (A) The cMWM and starting positions used in Experiment 1. (B) The cMWM used in Experiments 2 and 3, which replicates the environment developed by Hamilton and Sutherland (1999). 
Table 1

Design of Experiment 1

\begin{tabular}{cccc}
\hline & \multicolumn{2}{c}{ Acquisition } & \\
\cline { 2 - 3 } Group & Phase I & Phase II & Probes \\
\hline Blocking & $S_{1}{ }^{\text {D }}$ & $S_{1} \cup S_{2}{ }^{\mathrm{b}}$ & $S_{2}$ \\
Control & & $S_{1} \cup S_{2}$ & $S_{2}$ \\
\hline
\end{tabular}

Note-A standard blocking design was used to test whether a cognitive map will be updated when the corresponding environment is changed. During Phase I of acquisition, Cue Set $S_{1}$ was displayed. Then, during Phase II (compound training), another set, $S_{2}$, was added, such that no wall was devoid of cues. Finally, acquisition of $S_{2}$ was tested during the probe trials, in which $S_{1}$ was removed. Six different sets were used, each consisting of the cues from two walls. All possible set combinations were used for the blocking and control groups. The cues in each set could be in one of two spatial relations: They were on walls either (1) opposite each other (e.g., north-south) or (2) adjacent to each other (e.g., north-west). In order to obtain the same number of observations for each of these two cases, the number of subjects for the conditions in which the walls were adjacent to each other was half the number of subjects for walls opposite each other (i.e., 4 and 8, respectively). ${ }^{\mathrm{a}} S_{1} \in\{(\mathrm{N}, \mathrm{S}),(\mathrm{W}, \mathrm{E}),(\mathrm{N}, \mathrm{E})$, $(\mathrm{N}, \mathrm{W}),(\mathrm{S}, \mathrm{E}),(\mathrm{S}, \mathrm{W})\} . \quad{ }^{\mathrm{b}} S_{2} \in\{\mathrm{N}, \mathrm{E}, \mathrm{S}, \mathrm{W}\} \cap S_{1}$.

during the blocking group's compound training (see Table 1 for details). Thus, for the control group, these cues constituted a subset of the cues that had been available during all of acquisition. The subjects started both probe trials from the southernmost of the two starting positions in the southeast quadrant (Figure 1A).

The subjects were not informed that the target was removed prior to the search persistence probe (SPP), which therefore appeared to be similar to the preceding acquisition trials, with the exception that half of the walls were empty. This trial took $180 \mathrm{sec}$.

The SPP has traditionally been employed to measure knowledge of the target location, although it is not entirely clear to what extent the observed behavior reflects available spatial knowledge or current spatial performance. For example, over the course of acquisition, the subjects may have come to expect the target at a certain locationthat is, learned its position to a high degree of accuracy - and thus may have quickly started to look for it elsewhere once they realized during the probe trial that it could not be found at the usual place. Such behavior has often been interpreted, perhaps misleadingly, as indicating lack of place knowledge, whereas the observed behavior might, in fact, indicate precise knowledge of the target location. Hence, some studies have also analyzed the heading error during initial locomotion as another measure of place knowledge. To ameliorate this shortcoming of the standard probe trial, we developed an additional assessment method administered right after training the SPP: the location accuracy probe (LAP). The LAP minimizes the possibility that spatial strategy and performance - that is, expressed spatial behavior - will contaminate the assessment of spatial knowledge. Right before participating in this probe trial, the subjects were informed that the target had been absent in the preceding SPP and that it would also be absent on the following trial. The subjects were told to navigate to the exact spot at which they thought the target would be found if it were present. The subjects were aware that they had unlimited time available for this task. The subjects terminated the LAP by pressing the space bar. The design of the task and the instructions delivered immediately before the LAP started greatly reduced the probability that the subjects would recruit certain search strategies to quickly find the target, since (1) they knew why they were unable to find the target during the SPP, (2) they knew that the target could not be found in the LAP as well, and (3) they were clearly informed about the purpose of the test (measuring their location knowledge). It should be noted that during the immediately preceding SPP, the target could not be found and the platform location was not revealed, so new learning of the actual target location was not possible. Furthermore, no additional acquisition trial occurred between the SPP and the LAP, which, in this and the following ex- periments, were administered in sequence. Hence, behavior during the LAP necessarily relied on the spatial knowledge the subjects had acquired during the acquisition phase and, thus, appears to be a more valid test of available spatial knowledge than is the SPP.

\section{Measures}

The computer software administering the task recorded the time required to find the target (latency, in seconds) and the time spent in each quadrant during each trial (time in quadrant, in seconds).

SPP. Time in quadrant was used to analyze performance during this probe trial, which, unlike the acquisition trials, had the same duration for all the subjects, since the target could not be found. A performance index was computed for each subject. This index, $\tau$ (cf. Cheng \& Newcombe, 2005), expresses the relative time spent in the target quadrant $\left(p_{\mathrm{T}}\right)$ corrected for the time that would have been spent in the correct quadrant by chance alone $\left(p_{\mathrm{C}}=.25\right)$ :

$$
\tau=\frac{p_{\mathrm{T}}-p_{\mathrm{C}}}{1-p_{\mathrm{C}}}, \text { with } p_{\mathrm{T}}=\frac{t_{\mathrm{T}}}{t_{\mathrm{T}}+t_{\mathrm{O}}+t_{\mathrm{L}}+t_{\mathrm{R}}} .
$$

In addition to $\tau$, the time that was spent in each quadrant was considered to prevent misinterpretation of the index score. For example, if a subject distributed all available time in the SPP equally between the target and one other quadrant, $\tau$ would suggest good spatial knowledge, although, in fact, this individual was not able to decide which of the two quadrants was the correct one; in this case, the bimodal distribution is informative, since consideration of this search pattern suggests that critical spatial information permitting accurate localization of the target might not have been acquired by the subject, although sufficient knowledge was present to exclude half of the available space. In all the experiments reported here, however, no such bimodal distributions were observed, and, consequently, only $\tau$ is discussed.

LAP. This probe trial provided data to assess the subject's knowledge of the target location in the form of the shortest distance between the recalled and the actual target position $(\Delta T)$ - that is, the length of the line that connected the target's center with the position at which the subject stopped.

All the measures were taken in pixels. Charts of the recalled target positions were generated for exploratory purposes, in which average and dispersion were indicated in the form of an ellipse, the center of which denoted the arithmetic mean, and the major and minor axes represented the standard deviation of the $x$ - and $y$-coordinates of the recalled target positions, respectively.

\section{Results}

We predicted that both groups would (1) indistinguishably reduce the time required to find the target during acquisition, (2) predominantly search the target quadrant during the SPP, and (3) be equally able to recall the target's location in proximity to its actual position in the LAP. In short, no group differences were expected, thus establishing the absence of blocking.

The data from 1 subject were excluded because a software error rendered parts of the data file unusable.

In this and all the following experiments, the Type I error rate was set to $\alpha=.05$ for all the statistical analyses, $\eta^{2}$ was always computed for significant effects and interactions as a measure of effect size, and significant interactions were further analyzed with post hoc Scheffé tests.

\section{Acquisition}

The blocking group was exposed to twice as many acquisition trials as the control group. Therefore, only the first eight trials for both groups were considered. The change of latency over trials was analyzed with an $8 \times 2 \times$ $2 \times 2$ (trial $\times$ group $\times$ cue relation $\times$ sex $)$ repeated mea- 
A

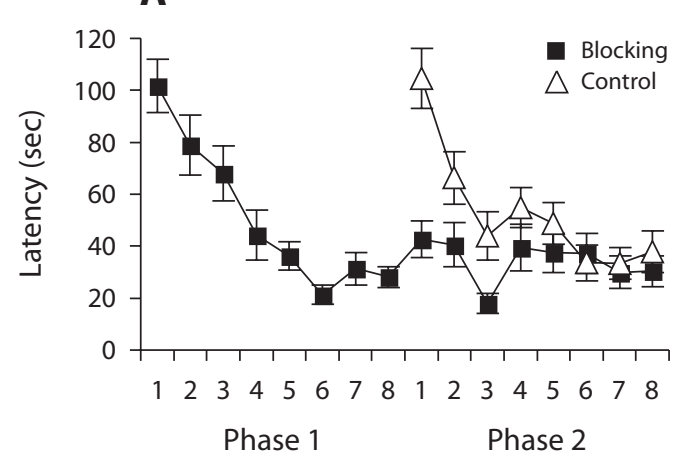

C

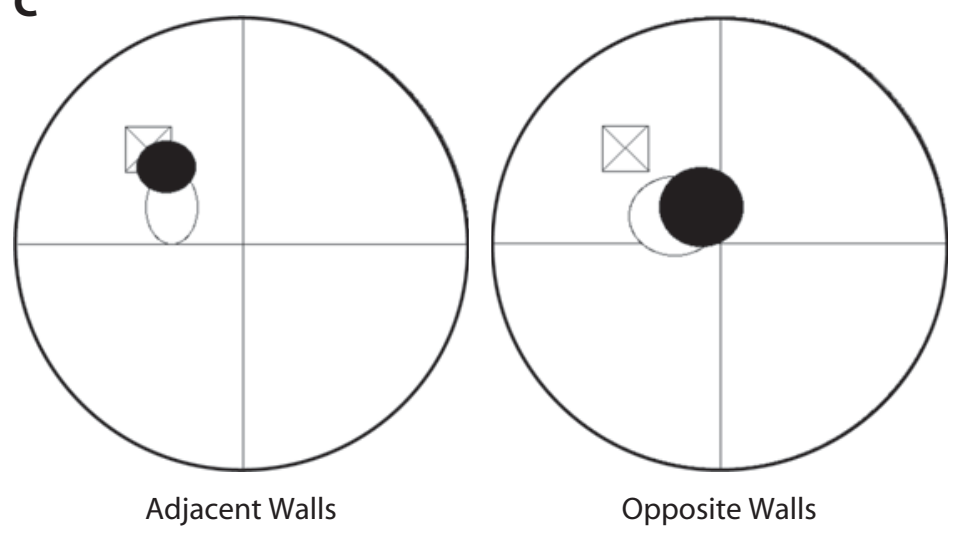

B
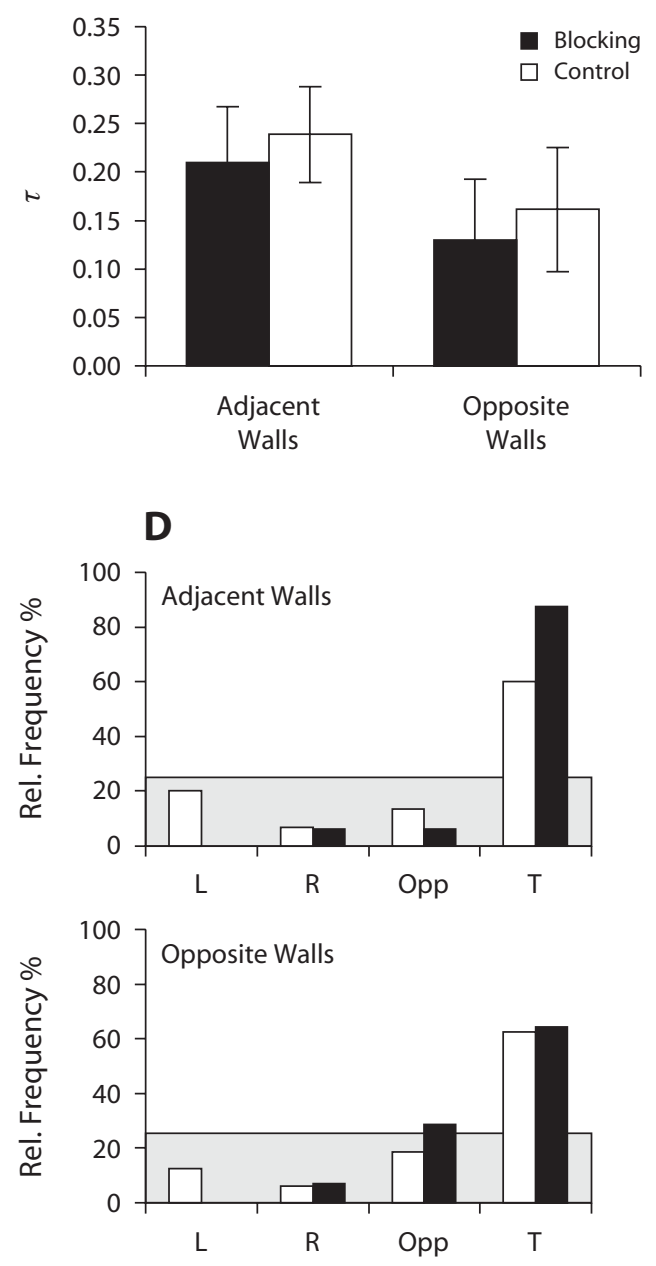

Figure 2. Results of Experiment 1: Absence of blocking in place learning. The control and blocking groups showed the same acquisition (A), and both indiscriminately searched the target quadrant during the search persistence probe, in which only the cues added during compound training were present (B). For the location accuracy probe, no group differences were found in accuracy recalling the target location (C) and in frequency with which the groups recalled the target in the correct quadrant (D; shaded area indicates performance at chance level). Performance during the probe trials was better when the available cues were on walls adjacent to each other than when they were on opposite walls. Error bars: \pm 1 standard error.

sures ANOVA, with trial as the repeated factor. Latency shortened significantly during acquisition $[F(1,385)=$ $\left.20.66, M S_{\mathrm{e}}=1,847.13, \eta^{2}=.27\right]$, and this main effect of trial was moderated neither by group $(F<1)$ nor by cue relation - that is, whether the cues in the cue sets were on walls opposite or adjacent to each other $(F<1)$. The male subjects were faster than the female ones $[F(1,55)=8.77$, $\left.M S_{\mathrm{e}}=4,633.15, \eta^{2}=.13\right]$. Interactions were not significant $(F<2.4)$. Post hoc tests determined that asymptote was reached after four trials in both groups and for both sexes. Introduction of the additional cues for the blocking group at the ninth trial was not accompanied by a change in latency (the 15-sec increase from the eighth to the ninth trial was not significant; $p>.99$ ).

Thus, the blocking and control groups reduced the time taken to find the target at about the same rate, relative to the trials in the acquisition phase (see Figure 2A).

\section{Probe Trials}

SPP. The time the subjects spent searching the target quadrant (see Figure $2 \mathrm{~B}$ ) was analyzed with a $2 \times 2 \times$ 2 (group $\times$ cue relation $\times$ sex) ANOVA on $\tau$. No group difference in preference for the correct quadrant was detected $(F<1)$, and male and female subjects searched the target quadrant equally intensively $(F<1)$. Whether cues were on walls adjacent to or opposite each other did not affect quadrant preference $(F<2)$. The interactions were not significant $(F<1)$.

In both groups and for both types of cue relations, the time spent in the correct quadrant was significantly above chance level, as multiple $t$ tests of $\tau$ against zero determined [control-adjacent, $t(62)=17.9, p<.01$; controlopposite, $t(62)=12.10, p<.01$, blocking-adjacent, $t(62)=15.75, p<.01$; blocking-opposite, $t(62)=9.75$, $p<.05]$. 
LAP. The frequency with which the target was recalled in the correct or in any other quadrant was analyzed with a chi-square test on a $2 \times 2$ (group $\times$ quadrant [target vs. other]) contingency table, separately for the conditions in which the cues were on walls adjacent to and opposite each other (see Figure 2D). Significant differences between the groups were not detected by either analysis; the subjects predominantly recalled the target in the correct quadrant, and they did so independent of the spatial relation among the cues [adjacent, $\chi^{2}(1)=3.06, p=.08$; opposite, $\left.\chi^{2}(1)=0.01, p=.92\right]$.

A $2 \times 2 \times 2($ group $\times$ cue relation $\times$ sex $)$ ANOVA on $\Delta T$ (see Figure 2C) revealed that both groups made an error of the same magnitude in recalling the target location and, thus, had comparable accuracy in their spatial knowledge of its place $(F<1)$. Sex differences were not observed $(F<1)$. Numerically, $\Delta T$ was smaller when cues were on walls adjacent to each other (difference of 20 pixels), but only a trend was observed for this effect $[F(1,53)=3.23, p=.08]$. None of the interactions were significant $(F<1)$.

Taken together, the results from the probe trials show the absence of a blocking effect and, thus, suggest that all groups learned the location of the target equally well.

\section{Discussion}

Locating the target was no more effective with cues that were present from the beginning of training than with cues that were added later on; the blocking and control groups predominantly searched the target during the probe trial in the correct area, indicating that they acquired the spatial relation between the available cues and the target. The subjects in both groups also made similar small errors in recalling the target location during the LAP, in which most of them recalled the target in the correct quadrant. In addition, the acquisition results show that performance of the blocking group was at asymptote when the new distal cues were introduced. Yet knowledge about these new cues was readily acquired. These results are consistent with cognitive map theory and support the conclusion that an allocentric representation was updated when the corresponding environment was modified; that is, blocking was absent in spatial learning. However, the results would not be readily expected on the basis of associative learning theories, which predict that such redundant cues should not be learned, especially when they cannot further improve performance.

\section{EXPERIMENT 2}

In a cognitive map, all available cues can be used for the purposes of orientation. This presumed property of cognitive maps has led to the use of random cue deletion controls as a way of demonstrating that subjects are indeed using such representations. Jacobs et al. (1998) conducted such a cue deletion study for the cMWM we used here. Performance degraded gracefully with the deletion of subsets of cues, and no particular subset deletion affected performance more than any other, thereby showing that cognitive maps were acquired in the task, in line with the absence of blocking we found here.
However, if navigation is impaired when some, but not other, distal cues have been removed after successful training, cognitive map theory supposes that behavior is being supported by egocentric representations. The assumption here is that specific cue-response and cue-reward pairings are strengthened during learning of egocentric representations and that this involves some, but not all, of the available cues. Whether this was also the case in the task used by Hamilton and Sutherland (1999) was addressed in this experiment.

We predicted that if subjects did not acquire a comprehensive allocentric representation of the environment and, instead, used egocentric representations, removal of a subset of cues would impair performance. We assumed that in the absence of a cognitive map, those cues in close proximity to the target would most likely be used, since navigation based on cues close to the target is easier and more accurate than navigation based on cues farther away.

\section{Method}

\section{Subjects}

One hundred twenty undergraduate students from the University of Arizona (18-45 years old, $M=20.4, S D=4.0 ; N_{\text {male }}=52 \approx$ $45 \%$ ) were recruited from the Psychology Department's subject pool and received course credit for participation.

\section{Apparatus}

MWM implementation. The CG Arena software (see Experiment 1) was used to replicate the computer-generated environment described by Hamilton and Sutherland (1999; hereafter, referred to as the original, whereas our version will be called the replication). The same computer system as that in Experiment 1 was used.

The dimensions of the original were stated in percentages (Hamilton \& Sutherland, 1999, p. 454, and Figure 1 on p. 455). Although these proportions were replicted exactly, it is likely that the actual dimensional values submitted to the CG Arena software were different (our parameters can be obtained upon request). Nevertheless, it took the subjects the same time to cross the original and replicated cMWMs (about $4 \mathrm{sec}$ ), and a full turn around the body axis also had the same duration (about $2.5 \mathrm{sec}$ )

The room's walls were colored in an irregular yellow-brownorange pattern (the yellow tone dominated the texture). Black lines indicated the horizontal and vertical corners of the walls. The ceiling was featureless gray, and the floor showed a tiled blue, irregularly patterned texture. The arena wall displayed a predominantly dark brown, irregular dot pattern. These visual features were almost indistinguishable from those used in the original. The original distal cues were used and positioned slightly off-center, as in the original (the graphic files, as well as the original software, were kindly supplied by Derek Hamilton upon request). Two cues were placed on each wall (see Figure 1B).

Movement was constrained to the circular arena. The arena wall prevented the subjects from looking over it when they were right in front of it. A bit farther away, however, the walls were fully visible. A pink square, the target, was hidden in the center of the arena's northeast quadrant. When the subject "stepped" on the target, it became visible, and a continuous clicking sound was played over the headphones. The subject's movements were then constrained to the target, so that free rotations around the body axis were still possible. After $30 \mathrm{sec}$ on the target, the trial terminated automatically; the subjects could, however, press the space bar to end the trial.

Movement was controlled as described in Experiment 1. Unlike in the original, the subjects could move backward (it was not possible to modify the default movement options of the CG Arena software).

The equivalence of replication and original was further subjectively controlled by running both implementations side by side and 
modifying the replication according to the observed differences until no such discrepancies were noticeable by two independent observers

\section{Procedure}

The subjects were randomly assigned to one of five groups, which had identical acquisition phases but varied with respect to the cues that were present during the probe trials (see Figure 3).

Spatial task. First, the subjects practiced moving with the arrow keys in an enivronment that was fundamentally different from the cMWM: An alpine scene was presented (snow-covered mountains and a partially clouded sky), in which the subjects could move in each direction without ever running into obstacles. The practice trial was self-paced, and the subjects could terminate it by pressing the space bar, which started the first acquisition trial. There was no such practice trial in the original.

As in the original, 32 acquisition trials were administered. The computer instructed the subjects to press the space bar to start the trial. Doing so, the subjects found themselves facing the arena wall. As in the original, subjects then had $1 \mathrm{~min}$ to find the target, after which the trial was automatically terminated. The subjects were trained with the same starting positions as those used in the original (north, east, south, and west), although the sequence of these positions most likely was different in the replication. The four possible positions were arranged in blocks (i.e., each block consisted of these and only these positions). The order of the positions within each block was pseudorandomly determined, and the final sequence was used for all the subjects. Administration of the possible starting positions in blocks eliminated variance resulting from the different distances to the target per starting point and, thus, allowed analysis of acquisition, using average latency per block.

The instructions that were presented prior to acquisition lacked, like the originals, information about (1) useful strategies, (2) the constancy of the target location and the varying starting positions, (3) the spatial relation between distal cues and target, and (4) the number, nature, and sequence of the experimental tasks. The instructions explained only how to move and that a practice trial would precede the actual task (see the Appendix for the actual instructions).

As in Experiment 1, knowledge of the target's location was assessed after the last acquisition trial in the SPP and LAP. During both probe trials, half of the distal cues were removed; which ones varied between groups (see Figure 3). The subjects were never informed about this modification.

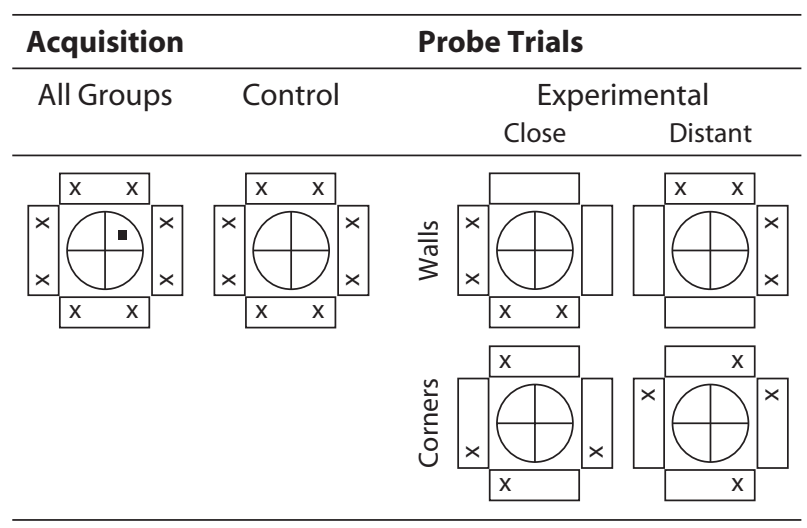

Figure 3. Design of Experiment 2. Eight distal cues, two at each wall, were present during the 32 acquisition trials. Groups differed with regard to the individual cues that were available during the 2 probe trials. No cues were removed for the control group, but in the close-corners and close-walls groups, four cues that were closer to the target were unavailable, whereas the equivalent, more distant cues were removed in the distant-corners and distant-walls groups.
After task completion, the subjects were asked whether they believed that the target remained in the same location during acquisition. This question was used as a possible indicator of place learning and acquisition of allocentric knowledge: The subjects would assume that the target's position was unpredictable only if they did not relate its location to distal cues.

Yes/no object recognition test. After the last probe trial, the subjects were presented with a self-paced computer-administered yes/no object recognition test for the distal cues that had been displayed on the walls of the cMWM. Feedback on accuracy was never provided so as not to bias the subjects' response strategies. The computer recorded responses and response latency (reaction time).

The subjects were presented with the distal cues and with the same number of lures, which were similar but had never been displayed before (for the stimuli used, see Figure A1 in the Appendix). The subjects were not informed about the ratio of old to new objects, but they were told that old and new objects would be presented. One object at a time was displayed, and the order in which old and new objects were shown was pseudorandomly determined for each subject. The subjects responded by clicking buttons with the computer mouse.

\section{Measures}

Place hypothesis. The subjects who believed that the target remained in the same place during acquisition were classified as having a constant place hypothesis; otherwise, they were classified as changing. This binary variable was used in all the analyses because it was assumed that those who deemed the target position to be unpredictable would not have related it to any distal cue and would, therefore, be outperformed by the subjects with a constant place hypothesis.

Object recognition test. Responses were classified as either a hit (an old item was identified as such), a miss (an old item was falsely judged as new), a false alarm (a new item was believed to be old), or a correct rejection (a new item was identified as new). Nonparametric indices for sensitivity and response bias were determined (two-threshold model; see Snodgrass \& Corwin, 1988). The corrected recognition score, $P_{\mathrm{r}}$, calculated by subtracting the false alarm probability $[p(\mathrm{FA})=(\# \mathrm{FA}) /(\#$ new items $)]$ from the hit probability $[p(\mathrm{H})=(\# \mathrm{H}) /(\#$ old items $)]$, could vary between -1.0 and 1.0. The corresponding response bias score, $B_{\mathrm{r}}$, was scaled to vary between 0 and 1 , with $B_{\mathrm{r}}>.5$ indicating liberal and $B_{\mathrm{r}}<.5$ indicating conservative biases:

$$
\begin{aligned}
P_{\mathrm{r}} & =p(\mathrm{H})-p(\mathrm{FA}) \\
B_{\mathrm{r}} & =\frac{p(\mathrm{FA})}{1-P_{\mathrm{r}}} .
\end{aligned}
$$

\section{Results}

Five subjects were excluded from the data set because they were taking prescription medication that potentially could interfere with task performance.

Seventy-two $(62 \%)$ of the subjects believed that the target remained in the same location during acquisition. A chi-square test on a two-way contingency table ( $\operatorname{sex} x$ place hypothesis) determined that the female subjects were almost twice as likely as the male subjects to suspect that the target had changed position $[p(\mathrm{~F})=.48>$ $p(\mathrm{M})=.25)]$.

\section{Acquisition}

Group differences in acquisition were not expected, and all the groups were predicted to reach the learning criterion by the end of training.

Change of latency during acquisition was analyzed with a $8 \times 5 \times 2 \times 2$ (trial block $\times$ group $\times$ sex $\times$ place hy- 


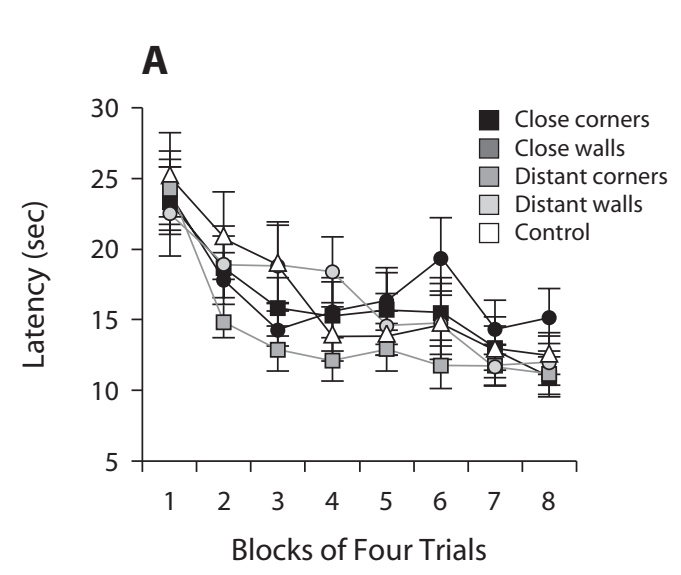

\section{B}
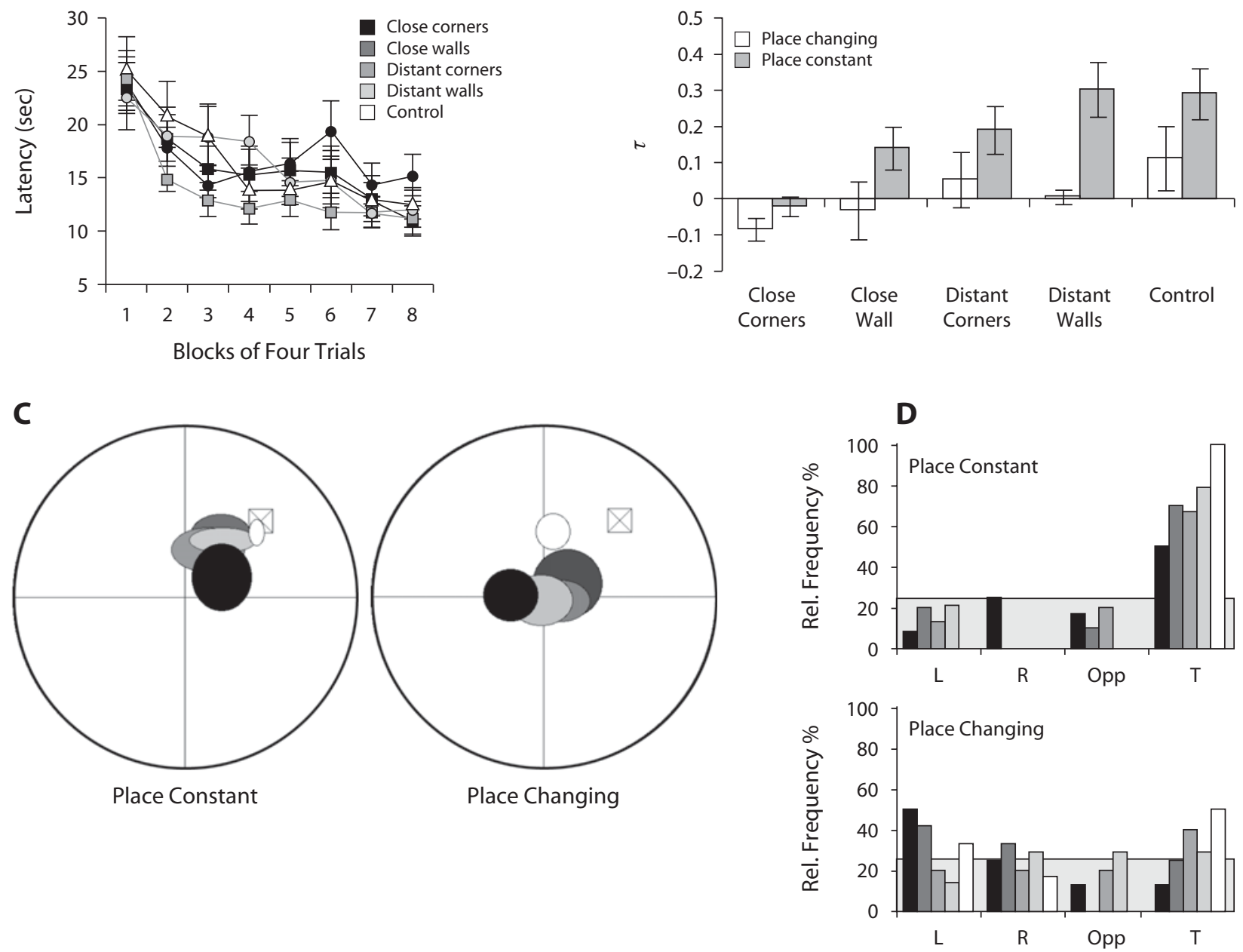

Figure 4. Results of Experiment 2. Only a small number of distal cues were used for place learning in the cMWM in which blocking was found (Hamilton \& Sutherland, 1999). All groups show the same acquisition (A), but subjects who lost cues in the corners close to the target searched the correct quadrant during the SPP at chance level (B), and only about half the subjects recalled its location in the correct quadrant in the LAP (C and D; shaded area indicates performance at chance level). Error bars: \pm 1 standard error.

pothesis) repeated measures ANOVA. Latency was found to significantly decrease over trials $[F(7,665)=19.39$, $\left.M S_{\mathrm{e}}=51.85, \eta^{2}=.20\right]$, and the reduction was the same for all the groups $(F<1$; see Figure 4A). Post hoc tests determined that the subjects reached asymptote by the third block. The effect of trial block on latency was moderated for later trials by place hypothesis $[F(7,665)=4.51$, $\left.M S_{\mathrm{e}}=51.85, \eta^{2}=.05\right]$, so that, independently of place hypothesis, the subjects improved their performance during the first two trial blocks but, then, only the subjects believing in a constant target location continued to do so. Finally, male subjects were faster than female subjects $\left[F(1,95)=8.63, M S_{\mathrm{e}}=433.47, \eta^{2}=.09\right]$. The remaining terms were not significant $(F<3)$.

\section{Probe Trials}

The control group was predicted to outperform all other groups, because no cues were removed for these subjects. It was expected that the subjects who lost cues close to the target (close corners and close walls) would perform worse than those who lost cues more distant (distant corners and distant walls).

SPP. Performance during this first probe trial, on which the subjects were not informed about the target's removal, was analyzed with a $5 \times 2 \times 2($ group $\times \operatorname{sex} \times$ place hypothesis) ANOVA on the relative time spent in the target quadrant $(\tau)$. A significant main effect of group $\left[F(4,95)=3.97, M S_{\mathrm{e}}=306.04, \eta^{2}=.17\right]$ was found, and post hoc tests revealed that the close-corners group, who lost the distal cues in the corner close to the target, was outperformed by the distant-walls and control groups (see Figure 4B). The subjects who suspected the target to change locations performed worse than those who believed otherwise $\left[F(1,95)=12.56, M S_{\mathrm{e}}=306.04, \eta^{2}=\right.$ .13]. Sex differences were not detected $(F<1)$, and the remaining terms were also nonsignificant $(F<1.4)$.

Multiple one-tailed $t$ tests compared $\tau$ against zero to determine whether the time spent in the correct quadrant 
was significantly above chance. In the absence of sex differences, the analysis was split by group and place hypothesis. The subjects with a changing place hypothesis searched the correct quadrant at chance level in all the groups $(.13<p<.99)$. Except for the close-corners group $[t(11)=-0.84, p=.79]$, the opposite was the case for the subjects with a constant place hypothesis [close walls, $t(11)=2.34, p<.05$; distant corners, $t(16)=2.89$, $p<.01$; distant walls, $t(15)=3.97, p<.01$; control, $t(14)=4.13, p<.01]$.

LAP. Data from 12 subjects were lost; 7 subjects prematurely terminated the trial by accidentally pressing the space bar before they had finished moving. For 3 subjects, it was impossible to unambiguously determine the recalled target location, given the path printout. Finally, 2 subjects did not understand the task instructions and searched the target as they did during the first probe trial. Despite the substantial data loss, the five groups still contained about the same number of observations, between 20 and 22 subjects.

The frequency with which the subjects recalled the target in either the correct or any other quadrant was analyzed with a chi-square test on a $5 \times 2$ (group $\times$ quadrant [target vs. other]) contingency table (see Figure 4D). Separate analyses were performed for each place hypothesis. The subjects with a changing place hypothesis recalled the target's location equally often in each quadrant $\left[\chi^{2}(4)=\right.$ $2.73, p=.60]$. The subjects who believed otherwise preferred the correct quadrant $\left[\chi^{2}(4)=9.13, p<.05\right]$. This effect was driven by the control and close-corners groups: As compared with the other groups, preference for the target quadrant was more pronounced in the former and less pronounced in the latter.

The error produced when the target location $(\Delta T)$ was recalled with a $5 \times 2 \times 2$ (group $\times$ sex $\times$ place hypothesis) ANOVA (see Figure 4C). The subjects with a constant place hypothesis were better than the subjects with a changing one $\left[F(1,83)=17.94, M S_{\mathrm{e}}=8,321.09\right.$, $\left.\eta^{2}=.22\right]$. A significant main effects of group was revealed $\left[F(4,83)=2.66, M S_{\mathrm{e}}=8,321.09, \eta^{2}=.13\right]$. Post hoc tests showed that the control group's error was smaller than that of the close-corners group. Other terms were not significant $(F<1)$.

\section{Object Recognition Test}

The ability to identify the distal cues was found to be rather poor $\left[p(\mathrm{H})=.44, p(\mathrm{FA})=.15, P_{\mathrm{r}}=.29, B_{\mathrm{r}}=.21\right]$. Recognition performance was analyzed in terms of the discrimination index $P_{\mathrm{r}}$ and the response bias index $B_{\mathrm{r}}$. A $5 \times 2 \times 2$ (group $\times$ sex $\times$ place hypothesis) ANOVA on $P_{\mathrm{r}}$ did not detect significant effects $(F<3)$. Multiple $t$ tests compared $P_{\mathrm{r}}$ against zero and established that recognition performance was above chance level in all the groups ( $p<.01$ in all groups). The corresponding analysis for $B_{\mathrm{r}}$ also showed no significant effects $(F<1.1)$. Multiple $t$ tests confirmed that in all the groups, $B_{\mathrm{r}}$ was significantly below $B_{\mathrm{r}}=.50$, the value indicating neutral bias ( $p<.01$ in all groups). The subjects thus exhibited a conservative bias.

\section{Discussion}

All the groups successfully learned the target location during acquisition, when all eight distal cues were present in the cMWM. However, removal of the cues close to the target during the subsequent two probe trials caused significant performance impairments: The subjects did not search the correct area for the target during the SPP, and their ability to recall the target position was impaired in the LAP. Thus, distal cues in the corner close to the target were predominantly recruited to encode the target's location. This finding suggests that a comprehensive cognitive map of the environment was not created and that, instead, performance reflected the use of egocentric representations.

The poor recognition of distal cues provides additional support for the conclusion that only some distal cues were used to guide navigation in this task. Apparently, only some of the available distal cues were explored and encoded. The remainder were not related to the target's position or to the location of those distal cues that were encoded; otherwise, removal of any subset of cues should have affected performance in the same way, and this clearly did not happen. Although cues in the corners were more likely to be used and encoded, it remains unclear precisely which cues were used, which were not used, and what precisely moderated the selection of cues in addition to cue location. One could speculate that some cues were more likely to be used because they were more salient and, hence, easier to remember than the others. Salience could depend on specific features of individual cues or on how cues compared with one another: Easily encoded cues would be most likely to guide spatial navigation. On this analysis, the blocking effect observed by Hamilton and Sutherland (1999) reflected specific properties of the particular distal cues that they used, rather than properties of cognitive map learning. Instead of falsifying the assumption of cognitive map theory that certain associative learning phenomena (e.g., blocking) are absent when cognitive maps are acquired, their result could indicate that spatial strategy choice depends on characteristics of available distal cues.

To conclude, our results suggest that Hamilton and Sutherland's (1999) blocking effect did not demonstrate a failure of cognitive map updating after novel distal cues were added to an environment; such maps might not have been created in the first place. Instead, it is possible that egocentric learning and egocentric navigational strategies were recruited and that blocking was obtained as a consequence. Rather than challenging cognitive map theory's automatic update assumption, these blocking results could show that, as predicted by cognitive map theory, egocentric spatial learning follows the rules of traditional associative learning, evincing phenomena such as blocking and overshadowing.

\section{EXPERIMENT 3}

A possible factor that could have moderated spatial strategy recruitment in the cMWM in which blocking was 
found is the nature of the task instructions, which did not encourage exploratory behavior and did not inform the subjects about the fact that the target position remained constant during the task. These neutral task instructions could have increased the likelihood that egocentric spatial strategies were predominantly recruited in Hamilton and Sutherland's (1999) paradigm. In contrast, the explicit instructions used in Experiment 1 might have predisposed the subjects to use allocentric, cognitive-map-based strategies.

We predicted that blocking would not be observed in the Hamilton and Sutherland (1999) apparatus when instructions favoring exploratory behavior were used, because cues added to the environment would be acquired under these conditions.

\section{Method}

\section{Subjects}

Forty-four undergraduate psychology students (18-22 years old, $M=19.3, S D=1.1 ; N_{\text {male }}=20 \approx 45 \%$ ) were recruited from the subject pool at the Psychology Department of the University of Arizona and received course credit for participation.

\section{Apparatus}

The cMWM from Experiment 2 was used (see Figure 1B), which replicated the environment in which blocking in human spatial learning had previously been observed (Hamilton \& Sutherland, 1999).

\section{Design}

As in the original blocking study by Hamilton and Sutherland (1999), two groups, blocking and control, were used to test whether previous training with a set of distal cues would block the acquisition of novel distal cues (see Table 2).

\section{Procedure}

Twenty-two subjects were randomly assigned to each group. The subjects first participated in the spatial task, which consisted of two acquisition phases for the blocking group and one phase for the control group, and then the SPP and LAP for both groups (see Table 2 for more details, and Figure 1B for the layout of the cMWM; individual trial parameters, such as duration, movement speed, etc., were as described in Experiment 2). Thus, the apparatus, spatial training, and first probe trial were exactly as in the original study by Hamilton and Sutherland (1999). Afterward, the subjects were asked whether they believed that the target had remained in the same position during acquisition; then recognition memory for the distal cues was assessed, as described in Experiment 2. Finally, recognition memory for the distal cues was assessed, as described in Experiment 2.

The subjects received the instructions used in Experiment 1. Unlike those used by Hamilton and Sutherland (1999) and in Experiment 2,

Table 2

Design of Experiment 3

\begin{tabular}{cccc}
\hline & \multicolumn{2}{c}{ Acquisition } & \\
\cline { 2 - 3 } Group & Phase I & Phase II & Probes \\
\hline Blocking & $A$ & $A+B$ & $B$ \\
Control & & $A+B$ & $B$ \\
\hline
\end{tabular}

Note-In the blocking group, subjects were exposed only to the four cues of Cue Set $A$ during the 20 trials of the first training phase. The four cues of Set $B$ were then added for the 12 trials of the second phase (compound training), and the ability to find the target with only these additional cues was tested in the subsequent probe trials, in which Cue Set $A$ was not present. The control group participated only in compound training but was, like the blocking group, exposed only to Cue Set $B$ during the probe trials. these instructions explicitly addressed the nature of the spatial task, stating that (1) the target would always remain in the same location, (2) the distal cues would be helpful in finding it, and (3) attending to the distal cues would therefore be beneficial (see Figure A2 in the Appendix for the text of the instructions used in our experiments).

\section{Results}

Although the subjects were informed about the constancy of the target location, some (7) nevertheless came to the conclusion that the opposite was the case. They were removed from the data set.

\section{Acquisition}

Differences between the blocking and control groups in acquisition performance were not expected. The results from the preceding experiments led to the prediction that the male subjects would find the target more quickly than would the female subjects.

Only the first three trial blocks were considered for each group (the control group was trained only for three blocks). A $2 \times 2 \times 3$ (group $\times$ sex $\times$ trial block) repeated measures ANOVA on latency, with trial block as the repeated factor, revealed that the subjects got faster during training $\left[F(2,66)=34.23, M S_{e}=52.80, \eta^{2}=.51\right]$ and that the male subjects outperformed the female subjects in that respect $\left[F(1,33)=16.41, M S_{\mathrm{e}}=182.56, \eta^{2}=.20\right]$. Group differences were not detected $(F<1.8$; see Figure 5A). The interactions were not significant $(F<2.3)$. Post hoc tests determined that the blocking group reached asymptote after the first three trial blocks.

\section{Probe Trials}

The absence of a blocking effect was predicted; the blocking and control group were expected (1) to spend most of their time searching the target in the correct quadrant during the SPP and (2) to recall the target's location at the same precision in the LAP.

SPP. A $2 \times 2$ ANOVA on $\tau$ detected that the control group spent significantly more time in the correct quadrant than did the blocking group $\left[F(1,33)=5.75, M S_{\mathrm{e}}=\right.$ $\left.376.58, \eta^{2}=.15\right]$. Although the main effect of sex was not significant $[F(1,33)=376.58, p=.22]$, the interaction between sex and group was $\left[F(1,33)=4.2, M S_{\mathrm{e}}=\right.$ $\left.376.58, \eta^{2}=.11\right]$. The group difference in overall performance was driven by the male subjects in the blocking group, who searched the target quadrant significantly less than did the female subjects $\left[F(1,33)=5.5, M S_{\mathrm{e}}=\right.$ $376.58]$. Such a difference was absent in the control group $(F<1$; see Figure 5B).

Multiple $t$ tests compared $\tau$ against zero to determine in what groups female and male subjects searched the target quadrant more extensively than would have been predicted by chance. Only the performance of the male subjects in the blocking group was at chance level [blocking-female, $t(10)=4.32, p<.01$; blocking-male, $t(7)=0.53, p=$ .62 ; control-female, $t(9)=3.09, p<.05$; control-male, $t(7)=11.07, p<.01]$. Thus, in the SPP, blocking was found only for the male subjects.

LAP. For both groups, the frequency of recalling the target in the correct quadrant was compared with the 
A

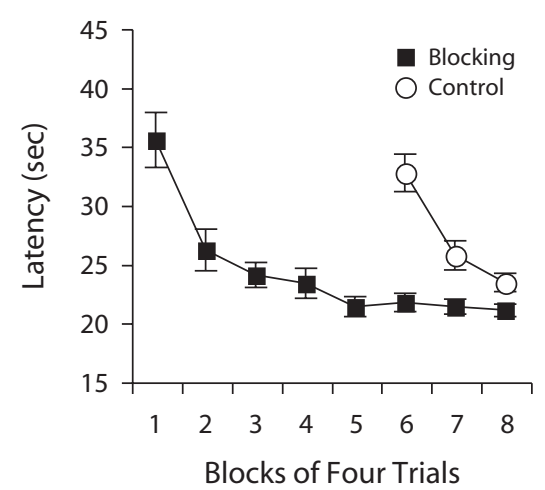

C

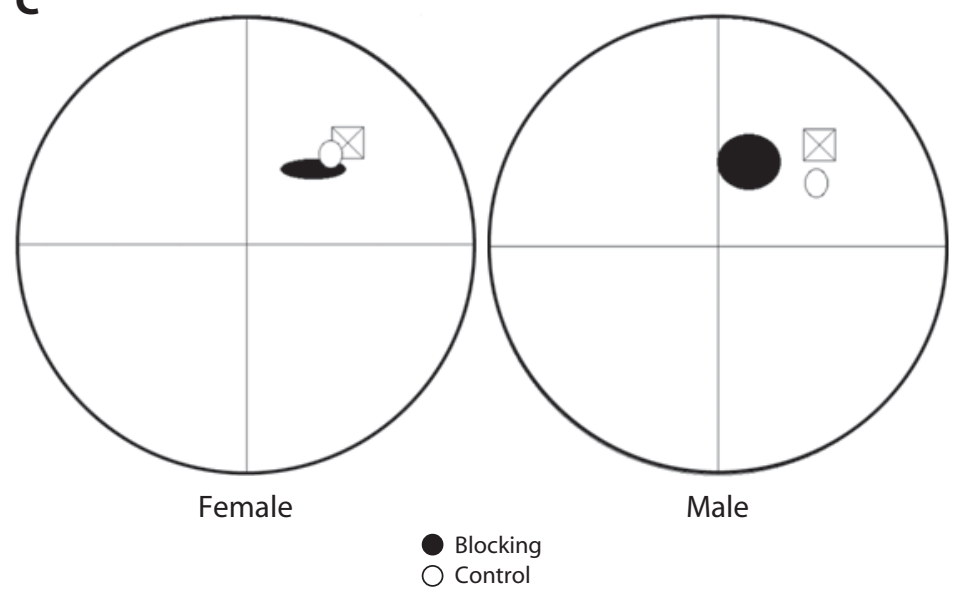

B

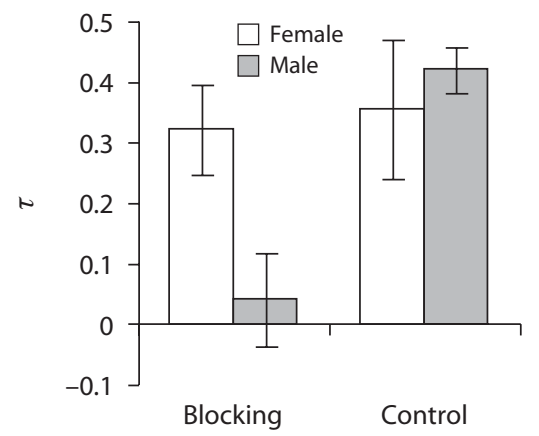

D
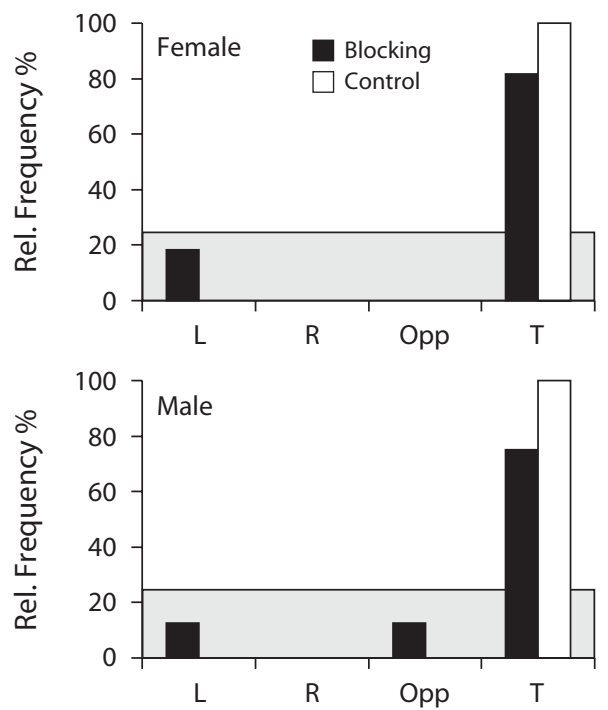

Figure 5. Results of Experiment 3: Instructions to explore attenuate blocking. The control and blocking groups show the same acquisition (A). During the SPP, blocking was observed only for male, but not female, subjects (B). This sex-specific blocking effect, however, was absent in the LAP, when the subjects were informed that the target was absent and were instructed to recall its position as precisely as possible. Although the male subjects made a larger error than did the female subjects in recalling the target's location, both sexes clearly recalled it in close proximity to its actual position (C), and both recalled it almost always in the correct quadrant (D; shaded area indicates performance at chance level). Error bars: \pm 1 standard error.

frequency of recalling it in any other quadrant (see Figure 5D). A chi-square test on the $2 \times 2$ (group $\times$ quadrant) contingency table did not detect group differences $\left(\chi^{2}=1.90, p=.17\right)$ : Most of the subjects in both groups recalled the target equally often in the correct quadrant. The blocking effect observed in the SPP was not found in this test of spatial knowledge.

However, a $2 \times 2$ (group $\times$ sex $)$ ANOVA on $\Delta T$ revealed that the blocking group produced a larger error in recalling the target position than did the control group $\left[F(1,33)=5.06, M S_{\mathrm{e}}=2,927.09, \eta^{2}=.13\right]$. Differences between the male and female subjects were not revealed $(F<1)$, and the interaction of both factors also was not significant $(F<1)$. Similar to the results for $\tau$, the larger error of the blocking group appeared to be caused primarily by the male subjects, whose distance to the target was about $25 \%$ larger than the distance produced by the female subjects in that group (see Figure 5C). However, despite the significant group difference, performance in both groups was good, so that blocking and control groups achieved short overall distances to the target, since, in both groups, the majority of the subjects recalled the target's location within its actual quadrant. This blocking effect thus reflected a reduction in the precision of place knowledge, rather than the absence of such knowledge overall.

\section{Object Recognition Test}

The subjects in both groups were predicted to recognize the distal cues in the yes/no recognition test equally well. No sex differences were expected.

First, the probability that an object would be correctly recognized as a distal cue $[p(\mathrm{H})]$ and the probability that a novel object would be falsely recognized as such $[p(\mathrm{FA})]$ were analyzed with a $2 \times 2 \times 2($ group $\times \operatorname{sex} \times$ response $)$ 
repeated measures ANOVA, with response as the repeated factor. The subjects were much more likely to produce hits than false alarms $\left[F(1,33)=270.70, M S_{\mathrm{e}}=0.12\right.$, $\left.\eta^{2}=.89\right]$. A trend was observed for the interaction of response and $\operatorname{sex}[F(1,33)=3.66, p=.06]$. It appeared that the male subjects were as likely as the female ones to falsely recognize a new object as an encountered distal cue but that the female subjects were about $10 \%$ more likely to produce a hit. No other effects were significant $(F<2.1)$.

The ability to correctly identify the initially available and later added distal cues was analyzed with a $2 \times 2 \times 2$ (group $\times$ sex $\times$ cue set) repeated measured ANOVA on hit probability, with cue set serving as the repeated factor. Hit probability was calculated for Cue Sets A and B separately for each subject $\left[p(\mathrm{H})_{\mathrm{A} / \mathrm{B}}=\# \mathrm{H}_{\mathrm{A} / \mathrm{B}} / \# \mathrm{Old}_{\mathrm{A} / \mathrm{B}}\right]$. The main effect of group was not significant $(F<1.2)$. The analysis revealed that the female subjects outperformed male subjects $\left[p\left(\mathrm{H}_{\mathrm{F}}\right)=.60>p\left(\mathrm{H}_{\mathrm{M}}\right)=.49 ; F(1,33)=\right.$ 4.42, $\left.M S_{\mathrm{e}}=0.04, \eta^{2}=.12\right]$. The hit probability was higher, overall, for Cue Set B than for A $\left[p\left(\mathrm{H}_{\mathrm{B}}\right)=.62>\right.$ $\left.p\left(\mathrm{H}_{\mathrm{A}}\right)=.48 ; F(1,33)=10.24, M S_{\mathrm{e}}=0.04, \eta^{2}=.24\right]$. The interactions were not significant $(F<2.8)$.

Whether the probability of correctly recognizing cues from the two sets was significantly above chance was analyzed with multiple one-tailed $t$ tests that compared $p(\mathrm{H})$ against .5. The analysis was split by cue set and sex. Cues from Set B were recognized above chance level by both sexes. For Cue Set A, this was the case only for the female subjects.

Finally, the discrimination index $P_{\mathrm{r}}$ and the response bias index $B_{\mathrm{r}}$ were analyzed separately. First, a $2 \times 2$ (group $\times$ sex) ANOVA was calculated for $P_{\mathrm{r}}$. Group differences were not detected $[F(1,33)=2.36, p=.14]$. A trend was revealed for $\operatorname{sex}[F(1,33)=3.66, p=.06]$, and the interaction between group and sex was not significant $(F<1)$. As was indicated in the obtained probabilities for hits and false alarms reported above, the female subjects were (numerically) better at discriminating novel objects from distal cues than were the male subjects. Despite this difference, multiple $t$ tests, which compared $P_{\mathrm{r}}$ against zero, revealed that performance was above chance for both the male and female subjects in both groups.

The corresponding analysis of $B_{\mathrm{r}}$ did not detect significant effects $(F<1)$. Overall, a rather conservative response bias was obtained $\left(B_{\mathrm{r}}=.19\right)$. Multiple $t$ tests confirmed this impression. In both groups, female and male subjects exhibited a bias significantly below .5 .

\section{Discussion}

Instructions that emphasized the utility of exploration attenuated blocking in our replication of the MWM in which this effect was originally found using different instructions (Hamilton \& Sutherland, 1999). The distal cues that were added during the second training phase were incorporated into the spatial representation of the environment, as performance during the second probe trial (LAP) demonstrated: Although the control group's distance to the actual target location was slightly smaller than that of the blocking group, both recalled the target position within the correct quadrant. This knowledge, however, was not expressed during the first probe trial (SPP). Here, the blocking group indeed spent less time searching the target in the correct quadrant, although both groups significantly preferred the target quadrant to the other quadrants. Further analysis of this effect revealed that in the blocking group, only the male subjects failed to prefer the target quadrant to the others; in fact, their performance was at chance level, whereas the female subjects in this group performed as well as the control group subjects. Thus, the comparatively small blocking effect is explained by male behavior alone.

The blocking and control groups learned to locate the target during training equally well, and the groups indistinguishably reduced the time required to do so. The subjects in the control group achieved a level of performance during three blocks of trials that was the same as that for the blocking group at the end of their eight blocks of training (asymptotic performance was reached after three blocks of four trials). As in the previous experiment, in both groups the male subjects located the target more quickly than did the female subjects.

Although the male subjects outperformed the female ones during acquisition, blocking was found only for the former: During the blocking group's SPP, the male subjects, but not the female ones, failed to selectively search the target quadrant, spending as much time there as they would have by chance.

This result could suggest that the male subjects did not integrate the additional cues into their spatial representation of the environment. However, LAP performance does not support this conclusion. During this test, for which the subjects were instructed to recall the target's location as precisely as possible, no blocking effect was observed. The vast majority of the subjects in both the blocking and control groups recalled the target in the correct quadrant, and the distance between the recalled and the actual target positions was small (in fact, it was as small as the average error produced in Experiment 2, in which the subjects navigated the same environment but received differentless informative-instructions; see Figure 4C). It should be noted that a significant albeit rather small group difference was observed for the error with which the target's location was recalled. However, in both groups, the vast majority of the subjects recalled the target position within the correct quadrant. The significant group difference for $\Delta T$ can, therefore, be interpreted as higher precision in the control group's knowledge of the target location, rather than as an absence of such knowledge in the blocking group (see Figure 5C).

Given the fact that learning about the target could not occur during the first probe trial (SPP), good performance in both groups during the subsequent probe (LAP) cannot be attributed to knowledge acquired in the SPP: If the male subjects in the blocking group had failed to form an association between the distal cues added during compound training and the target location, they should not have been able to locate the target during either the SPP or the LAP. Despite the lack of additional training between these probe 
trials, the male subjects in both the blocking and control groups recalled the target location with high precision during the LAP. The spatial knowledge expressed during the LAP must also have been available during the SPP but, for some reason, was not used. That is, the blocking effect observed in the male subjects in this experiment can best be characterized as an effect on the expression of spatial knowledge, and not on its acquisition. Since the critical assumption of cognitive map theory being put to test was whether automatic updating of allocentric spatial representations would occur if the environment changed - that is, whether new distal cues would be integrated into an existing allocentric spatial representation-our results seem to confirm the automatic updating assumption. Given this, why was this knowledge not expressed under all the testing conditions?

The recognition data indicate that the additional distal cues were not ignored. Neither sex nor group differences were observed for the ability to discriminate old from new objects, although the female subjects were better than the male ones. More important, the later added cues (Set B), which were the only ones available during the probe trials, were recognized above chance level by the male and female subjects alike. Interestingly, although the subjects in the blocking group were exposed to Cue Set A for 32 trials, overall recognition performance was rather low, especially given the task instructions that explicitly mentioned the utility of the distal cues. This might indicate that it was hard to encode the cues, which were mostly pictures of a rather abstract nature (see Figure 1B).

The higher persistence with which the female subjects in the blocking group searched the target quadrant during the SPP could indicate that the male subjects in this group had more confidence in their spatial knowledge. Thus, when they were not able to find the target after briefly searching its actual location, the male subjects may have concluded that it had moved and may have gone looking for it someplace else. Although we have no data with which to compare initial and late spatial navigation during the SPP, the acquisition curve for the male subjects in the blocking group provides some support for this interpretation. In this group, the male subjects reached asymptote within the first trial block, whereas the female subjects continually improved their performance - at least numerically - until the last block. At the end of eight blocks, of which seven (i.e., 28 trials) were at asymptote, the male subjects were accustomed to quickly finding the target at a specific location. In contrast, the male subjects in the control group reached asymptotic performance at the end of the three-block acquisition phase and thus were not trained enough at asymptote to reach the same level of confidence in their spatial knowledge as the male subjects in the blocking group. As a consequence, they searched the target quadrant more persistently than did their male counterparts in the blocking group, because they were not prepared to rapidly conclude from their inability to find the target that it had moved to a different location.

This account further implies either that the male subjects had only partly encoded the task instructions that were presented to them before acquisition and that ex- plicitly stated that the target would always be in the same place, or that they were ignoring or had forgotten them by the time they reached the first probe trial. By contrast, female behavior during the first probe trial was in compliance with the instructions. At the end of the first probe trial, all the subjects were informed of the target's absence and were told that this would also be the case in the ensuing LAP. This reminder of the original instructions apparently sufficed to produce the requested behavior in the male subjects.

The learning curve for the male subjects in the blocking group also permits another interpretation for the possible mechanism moderating the dissociation of SPP and LAP performance. Packard and McGaugh (1996) showed that extensive training in rats may lead to a shift from allocentric to egocentric behavior. They further demonstrated that this type of shift does not reflect loss of allocentric knowledge; rather, this knowledge is no longer expressed but remains intact and accessible. A similar shift might have occurred in the male subjects in our study: Their learning curve might indicate that they received overtraining, which could have led to a preference for egocentric over allocentric behavior. As a consequence, a blocking effect was observed during the SPP. However, after they were informed about the absence of the target prior to the LAP, they again used their available allocentric knowledge, and, as a consequence, the blocking effect was attenuated.

To conclude, it is unlikely that the divergent probe trial results for the male subjects in the blocking group were due to discrepancies in spatial knowledge; instead, they were due to differences in its expression.

\section{GENERAL DISCUSSION}

We found that blocking is not observed in a cMWM in which subjects create allocentric representations (Experiment 1). Our results show that subjects integrate newly added cues into an already highly predictive cognitive map. Furthermore, we found that such comprehensive allocentric representations are not generated in a different computer implementation of the task (Experiment 2), in which others had found blocking (Hamilton \& Sutherland, 1999). In their variant of the water maze, subjects predominantly associated the target location with a small number of cues - specifically, those closer to the target - so that the subjects' ability to find the target was compromised when these cues were removed. Such an effect would not be observed if a comprehensive cognitive map guided behavior, since, by definition, a map permits navigation when any subset of distal cues is available. The blocking effect that was observed in this MWM variant could thus be due to the fact that the subjects were more likely to recruit spatial strategies that led to only partial mapping in this specific version of the task.

According to cognitive map theory, exploratory behavior is required for map learning, and the relationship of spatial memory and performance and exploratory behavior has been demonstrated in hippocampus-dependent tasks, such as contextual fear conditioning (McHugh \& Tonegawa, 2007) and the MWM (e.g., Schulz, Kouri, \& 
Huston, 2007). Further providing support for the role of exploration in allocentric learning, the MWM variant in which we failed to find blocking (Experiment 1) employed instructions that emphasized the utility of exploratory behavior for successful performance, whereas such information was omitted in the instructions for the MWM implementation in which blocking was found (Hamilton \& Sutherland, 1999) and in which cognitive maps of the environment were not created (Experiment 2). Indeed, when the former instructions were provided instead of the latter, blocking was attenuated in the replication of the computer implementation of the MWM in which it was originally detected (Experiment 3): Male subjects, but not female ones, showed a blocking effect during the first probe trial, when the subjects were not aware that the target had been removed. However, male performance recovered during the immediately following second probe trial, prior to which the subjects were informed that they were not able to find the target and were asked to navigate to the target's former location as precisely as possible. Since the subjects were not able to relearn the target location before the second probe trial, the absence of blocking in the second trial suggests that the blocking effect in the first probe trial reflected a failure to express existing knowledge.

This interpretation of our data, however, appears to be in conflict with a study in which blocking was observed in rats despite apparent exploration of a newly added landmark (Biegler \& Morris, 1999). Although this study appears to be in opposition to our results, it is possible that the blocking effect Biegler and Morris obtained is similar in nature to the blocking effect we report in Experiment 3, in which one procedure probing spatial knowledge (SPP) yielded results indicating blocking, but another did not (LAP). It is possible that the rats in Biegler and Morris's experiment incorporated the explored landmark into their allocentric representation of the environment but failed to express it during the spatial test, for reasons similar to those that may have caused our male human subjects to initially exhibit blocking. In both studies, the subjects were extensively trained, which might have favored recruitment of egocentric behavior at a later point in the experiment (cf. Packard \& McGaugh, 1996). Allocentric behavior might have been dominant during compound training, when the additional spatial cues were introduced, and exploratory behavior was consequently observed. However, when spatial knowledge was tested after further training, egocentric strategies controlled behavior, and blocking was observed. Lacking empirical data, these ideas remain speculative and suggest further research in which, for example, the relation between training intensity and blocking effects will be systematically studied.

In summary, our results suggest that, at least in our experiments, blocking effects in spatial learning do not demonstrate a failure to acquire novel spatial knowledge during compound training. Rather, as cognitive map theory predicts, when behavior is controlled by allocentric strategies, spatial representations are automatically updated after exploration of novel cues. Although we interpret our data within the framework of cognitive map theory, from an associative learning perspective, our results lend sup- port to models proposing that blocking represents a performance (i.e., retrieval; see, e.g., Denniston, Savastano, $\&$ Miller, 2001), rather than a learning, deficit (e.g., Rescorla \& Wagner, 1972).

\section{Sex Differences}

In our experiments, male subjects were faster than female ones in locating the target during acquisition. This finding replicates earlier results, in which the same male performance advantage was discovered for humans in computer-simulated allocentric tasks (Astur, Ortiz, \& Sutherland, 1998). We would, however, like to suggest that this performance advantage for male subjects does not indicate differences in the spatial knowledge that is acquired during training. First, male and female subjects either searched the target in the correct quadrant or failed to do so equally often, and they produced comparable errors when asked to recall the target's location (but see Astur et al., 1998, who found the opposite). Second, in Experiment 3, the female subjects in the blocking group searched the target predominantly in the correct area during the SPP trial, whereas male preference for the correct quadrant was at chance level. However, when the subjects were aware that they would not be able to find the target during the subsequent LAP, both sexes recalled the target predominantly in the correct quadrant and in close proximity to its actual location. As was pointed out above, spatial knowledge about target location could not be acquired between the first and second probe trials, since interpolated training trials were not administered, and the target location was never revealed during or after the first probe trial. Although the same spatial knowledge was available during both probe trials, the male subjects expressed their knowledge differently in the two trials.

There are two possible explanations for this dissociation of performance in the male subjects in Experiment 3. First, male subjects simply may not follow task instructions as compliantly as do female subjects, or they may not pay as much attention to these instructions as do female subjects. This is a plausible explanation, because the subjects were informed at the beginning that the target would remain in the same location and were again reminded of this fact at the beginning of the second probe trial, during which male knowledge about the target location recovered.

The second explanation focuses on differences in strategic behavior. The important result is that there were no large sex differences in this experiment for knowledge of the target location; this is the implication of the good performance of both male and female subjects during the LAP. Since performance differences during the first probe trial are unlikely to indicate spatial knowledge differences, our results appear to demonstrate sex differences in spatial strategy choice or in the interpretation of behavioral outcome. This interpretation, however, cannot be verified with the available data and remains speculative. It is, for example, impossible to analyze male performance during the first half of the SPP using the current version of our software, which could indicate whether the male subjects initially tried to find the target in the correct quadrant but quickly decided that it was not in that location. 
In general, sex differences in spatial abilities are rather unreliable (Caplan, MacPherson, \& Tobin, 1985). The most robust difference between male and female subjects has been documented for mental rotation tasks (see Astur, Tropp, Sava, Constable, \& Markus, 2004), but even here divergent findings have been reported (Caplan et al., 1985). In spatial navigation, female subjects have been shown to preferentially recruit landmarks for orientation, whereas male subjects are thought to additionally employ cardinal coordinates and geometry (e.g., Sandstrom, Kaufman, \& Huettel, 1998). But this does not imply that female subjects are incapable of using geometry. Rather, for unknown reasons, male and female subjects are more likely to apply one spatial strategy than to apply another, and this appears to be moderated by spatial task affordances (Astur et al., 2004). Further research is clearly needed to explore these issues in a systematic way.

\section{Methodological Issues}

Traditionally, performance in the MWM has been quantified in terms of the reduction of latency during acquisition and the time spent in the quadrants of the circular tank during the standard probe trial, during which the target is removed from the maze (e.g., Astur et al., 1998; Hamilton \& Sutherland, 1999; Jacobs et al., 1997; Morris, 1981). The former is used to measure learning, whereas the latter is interpreted as a measure of spatial knowledge. It is, however, not entirely clear what the standard probe trial really measures - whether it indexes place knowledge, spatial strategy, or potentially pathological forms of perseverance. We developed the LAP to more reliably measure spatial knowledge. Subjects are informed about the absence of the target and are instructed to recall the learned target location as precisely as possible by navigating to its assumed location in the maze. The utility of this task was clearly demonstrated in Experiment 3, in which the traditional probe trial indicated a sex difference in spatial knowledge, which could not be detected by the LAP. Since the LAP trial immediately follows the standard probe trial, the target location cannot be learned between these two tests, and differences in performance cannot be attributed to differences in spatial knowledge; rather, they indicate differences in the expression of such knowledge.

The spatial strategies employed by animals are analyzed by interpreting their search paths, and this is often also the case in human studies using the MWM. As an alternative method for assessment of human spatial behavior, we found that asking subjects whether they believed that the target's location had varied during acquisition was very effective. Those who came to believe that the target location remained unstable never acquired its location, which is trivial but, nevertheless, explained about half the variance in all the dependent variables.

\section{Conclusion}

Blocking has occasionally been observed in allocentric tasks, suggesting to some that allocentric learning employs associative learning mechanisms as well, contrary to the predictions of cognitive map theory (e.g., Biegler \& Morris, 1999; Chamizo et al., 1985; Hamilton \&
Sutherland, 1999; Rodrigo et al., 1997; Sanchez-Moreno et al., 1999). In most of these studies, however, direct evidence about what kind of spatial representations were acquired during the course of learning was not provided (but see Blaisdell \& Cook, 2005; Chamizo, Rodrigo, \& Mackintosh, 2006; and Sawa, Leising, \& Blaisdell, 2005, for studies suggesting the presence of associative mechanisms mediating learning of allocentric representations). Therefore, it is possible that subjects recruited egocentric strategies or that they shifted in the course of training from allocentric to egocentric strategies. If that were the case, the blocking findings do not challenge cognitive map theory's assumption that map learning follows different rules than does egocentric spatial learning. Indeed, these findings would be consistent with the theory, since associative learning phenomena are predicted for egocentric learning. Absent an independent demonstration of what kind of spatial strategies were recruited to produce spatial behavior, it is not entirely clear what the results indicate.

The absence of blocking observed in the present set of experiments supports cognitive map theory, but the theory does not explain why the presence or absence of blocking should depend on specific instructions. Since cognitive map theory assumes that subjects default to cognitive map learning in allocentric tasks, the fact that specific instructions are required to promote exploratory behavior and map updating is not readily understood. Cognitive map theory does provide some hints; for example, it proposes that distal and not proximal cues will become parts of maps. However, what qualifies as a distal cue is not clearly defined. It also proposes that stable features of the environment are most likely to be represented in internal maps, such as geometric information. The results from our studies suggest that the abstract cues present in Hamilton and Sutherland's (1999) task may not be suitable for cognitive map learning: The poor object recognition scores for these cues, whether exploration was (Experiment 3) or was not (Experiment 2) encouraged, point to the possibility that they were hard to encode, despite extensive training and cue exposure. It would be interesting to study whether map updating is moderated by the quality of the distal cues added to the environment; abstract cues, such as those predominantly used by Hamilton and Sutherland, might lead to different results than do concrete cues, which were used in Experiment 1. In this context, it should be noted that the cues close to the target in the Hamilton and Sutherland task were among the small number of rather concrete images (see the Appendix, Figure A1) and that removal of these cues led to impaired performance in Experiment 2.

As we have pointed out elsewhere (Nadel \& Hardt, 2004), in most spatial tasks, organisms have a choice: They can use either egocentric or allocentric strategies, and they might even switch from one strategy to another in the course of the experiment. Demonstrations of correlations between spatial strategy and brain activity support the notion of multiple memory systems that exert variable control over behavior in response to specific environmental parameters (e.g., Bohbot, Iaria, \& Petrides, 2004; Eichenbaum, Stewart, \& Morris, 1990; Harvey 
et al., 2008; Iaria, Petrides, Dagher, Pike, \& Bohbot, 2003; Nadel \& O'Keefe, 1974; O'Keefe \& Nadel, 1978; Packard \& McGaugh, 1996; Sherry \& Schacter, 1987; White \& McDonald, 2002). It appears that subtle environmental changes can cause a shift from one cognitive strategy to another in solving the same problem. Future studies should explore the parameters that determine what kind of spatial strategy is recruited to solve specific problems.

\section{AUTHOR NOTE}

This study was part of the first author's dissertation at the University of Arizona and was supported by grants to the Cognitive Neuroscience Center from the Flinn Foundation and the McDonnell-Pew Program. We thank W. Jake Jacobs, Alfred Kaszniak, Elizabeth Glisky, and Lee Ryan for their insightful comments on earlier versions of the manuscript. Correspondence concerning this article should be addressed to O. Hardt, Department of Psychology, McGill University, 1205 Dr. Penfield Ave., Montréal, QC, H3A 1B1 Canada (e-mail: oliver.hardt@mac.com).

\section{REFERENCES}

Astur, R. S., Ortiz, M. L., \& Sutherland, R. J. (1998). A characterization of performance by men and women in a virtual Morris water task: A large and reliable sex difference. Behavioural Brain Research, 93, $185-190$

Astur, R. S., Tropp, J., Sava, S., Constable, R. T., \& Markus, E. J. (2004). Sex differences and correlations in a virtual Morris water task, a virtual radial arm maze, and mental rotation. Behavioural Brain Research, 151, 103-115.

BIEGLER, R., \& Morris, R. G. M. (1999). Blocking in the spatial domain with arrays of discrete landmarks. Journal of Experimental Psychology: Animal Behavior Processes, 25, 334-351.

BlaISDELL, A. P., \& COOK, R. G. (2005). Integration of spatial maps in pigeons. Animal Cognition, 8, 7-16.

Bohbot, V. D., Iaria, G., \& Petrides, M. (2004). Hippocampal function and spatial memory: Evidence from functional neuroimaging in normal healthy subjects and patients with medial temporal lobe resections. Neuropsychology, 18, 418-425.

Brown, M. F., Yang, S. Y., \& DiGian, K. A. (2002). No evidence for overshadowing or facilitation of spatial pattern learning by visual cues. Animal Learning \& Behavior, 30, 363-375.

Caplan, P. J., MacPherson, G. M., \& Tobin, P. (1985). Do sexrelated differences in spatial ability exist? American Psychologist, 40, 786-799.

Chamizo, V. D., Rodrigo, T., \& Mackintosh, N. J. (2006). Spatial integration with rats. Leaning \& Behavior, 34, 348-354.

Chamizo, V. D., Sterio, D., \& Mackintosh, N. J. (1985). Blocking and overshadowing between intra-maze and extra-maze cues: A test of the independence of locale and guidance learning. Quarterly Journal of Experimental Psychology, 37B, 235-253.

Cheng, K., \& Newcombe, N. S. (2005). Is there a geometric module for spatial orientation? Squaring theory and evidence. Psychonomic Bulletin \& Review, 12, 1-23.

Denniston, J. C., Savastano, H. I., \& Miller, R. R. (2001). The extended comparator hypothesis: Learning by contiguity, responding by relative strength. In R. R. Mowrer \& S. B. Klein (Eds.), Handbook of contemporary learning theories (pp. 65-117). Mahwah, NJ: Erlbaum.

Doeller, C. F., \& Burgess, N. (2008). Distinct error-correcting and incidental learning of location relative to landmarks and boundaries. Proceedings of the National Academy of Sciences, 105, 5909-5914.

Eichenbaum, H., Stewart, C., \& Morris, R. G. M. (1990). Hippocampal representation in place learning. Journal of Neuroscience, 10, 3531-3542.

Graham, M., Good, M. A., McGregor, A., \& Pearce, J. M. (2006). Spatial learning based on the shape of the environment is influenced by properties of the objects forming the shape. Journal of Experimental Psychology: Animal Behavior Processes, 32, 44-59.

Gray, E. R., Bloomfield, L. L., Ferrey, A., Spetch, M. L., \& Sturdy, C. B. (2005). Spatial encoding in mountain chickadees: Features overshadow geometry. Biology Letters, 1, 314-317.
Hamilton, D. A., \& Sutherland, R. J. (1999). Blocking in human place learning: Evidence from virtual navigation. Psychobiology, 27, 453-461.

Harvey, D. R., McGauran, A.-M. T., Murphy, J., Burns, L., MCMonagle, E., \& Commins, S. (2008). Emergence of an egocentric cue guiding and allocentric inferring strategy that mirrors hippocampal brain-derived neurotrophic factor (BDNF) expression in the Morris water maze. Neurobiology of Learning \& Memory, 89, 462-479.

Hayward, A., Good, M. A., \& Pearce, J. M. (2004). Failure of a landmark to restrict spatial learning based on the shape of the environment. Quarterly Journal of Experimental Psychology, 57B, 289-314.

Hayward, A., McGregor, A., Good, M. A., \& Pearce, J. M. (2003). Absence of overshadowing and blocking between landmarks and the geometric cues provided by the shape of the test arena. Quarterly Journal of Experimental Psychology, 56B, 114-126.

iaria, G., Petrides, M., Dagher, A., Pike, B., \& Bohbot, V. D. (2003). Cognitive strategies dependent on the hippocampus and caudate nucleus in human navigation: Variability and change with practice. Journal of Neuroscience, 23, 5945-5952.

Jacobs, W. J., Laurance, H. E., \& Thomas, K. G. F. (1997). Place learning in virtual space I: Acquisition, overshadowing, and transfer. Learning \& Motivation, 28, 521-541.

Jacobs, W. J., Thomas, K. G. F., Laurance, H. E., \& Nadel, L. (1998). Place learning in virtual space: II. Topographical relations as one dimension of stimulus control. Learning \& Motivation, 29, 288-308.

KAMIN, L. J. (1969). Predictability, surprise, attention, and conditioning. In B. A. Campbell \& R. M. Church (Eds.), Punishment and aversive behavior (pp. 276-296). New York: Appleton-Century-Crofts.

Mackintosh, N. J. (1975). A theory of attention: Variations in the associability of stimuli with reinforcement. Psychological Review, 82, 276-298.

McGregor, A., Hayward, A. J., Pearce, J. M., \& Good, M. A. (2004). Hippocampal lesions disrupt navigation based on the shape of the environment. Behavioral Neuroscience, 118, 1011-1021.

McHugh, T. J., \& Tonegawa, S. (2007). Spatial exploration is required for the formation of contextual fear memory. Behavioral Neuroscience, 121, 335-339.

MilleR, N. Y., \& Shettleworth, S. J. (2007). Learning about environmental geometry: An associative model. Journal of Experimental Psychology: Animal Behavior Processes, 33, 191-212.

Morris, R. G. M. (1981). Spatial localisation does not require the presence of local cues. Learning \& Motivation, 12, 239-260.

NAdEL, L., \& HARDT, O. (2004). The spatial brain. Neuropsychology, 18, 473-476.

Nadel, L., \& O'Keefe, J. (1974). The hippocampus in pieces and patches: An essay on modes of explanation on physiological psychology. In R. Bellairs \& E. G. Gray (Eds.), Essays on the nervous system: A festschrift for J. Z. Young (pp. 367-390). Oxford: Oxford University Press, Clarendon Press.

NAdel, L., \& Willner, J. (1980). Context and conditioning: A place for space. Physiological Psychology, 8, 218-228.

O'KeEFE, J., \& NADEL, L. (1978). The hippocampus as a cognitive map. Oxford: Oxford University Press, Clarendon Press.

PaCKard, M. G., \& McGaUgh, J. L. (1996). Inactivation of the hippocampus or caudate nucleus with lidocaine differentially affects expression or place response learning. Neurobiology of Learning \& Memory, 65, 65-72.

Pearce, J. M., Good, M. A., Jones, P. M., \& McGregor, A. (2004). Transfer of spatial behavior between different environments: Implications for theories of spatial learning and for the role of the hippocampus in spatial learning. Journal of Experimental Psychology: Animal Behavior Processes, 30, 135-147.

Pearce, J. M., Ward-Robinson, J., Good, M., Fussell, C., \& Aydin, A. (2001). Influence of a beacon on spatial learning based on the shape of the test environment. Journal of Experimental Psychology: Animal Behavior Processes, 27, 329-344.

Rescorla, R. A., \& WaGner, A. R. (1972). A theory of Pavlovian conditioning: Variations in the effectiveness of reinforcement and nonreinforcement. In A. H. Black \& W. F. Prokasy (Eds.), Classical conditioning II: Current research and theory (pp. 64-99). New York: Appleton-Century-Crofts.

Rodrigo, T., Chamizo, V. D., McLaren, I. P. L., \& Mackintosh, N. J. 
(1997). Blocking in the spatial domain. Journal of Experimental Psychology: Animal Behavior Processes, 23, 110-118.

Sanchez-Moreno, J., Rodrigo, T., Chamizo, V. D., \& Mackintosh, N. J. (1999). Overshadowing in the spatial domain. Animal Learning \& Behavior, 27, 391-398.

Sandstrom, N. L., Kaufman, J., \& Huettel, S. A. (1998). Males and females use different distal cues in a virtual environment navigation task. Cognitive Brain Research, 6, 351-360.

Sawa, K., Leising, K. J., \& Blaisdell, A. P. (2005). Sensory preconditioning in spatial learning using a touch screen task in pigeons. Journal of Experimental Psychology: Animal Behavior Processes, 31, 368-375.

Schulz, D., Kouri, C., \& Huston, J. P. (2007). Behavior on the water maze platform: Relationship to learning and open field exploration in aged and adult rats. Brain Research Bulletin, 74, 206-215.

Sherry, D. F., \& Schacter, D. L. (1987). The evolution of multiple memory systems. Psychological Review, 94, 439-454.

Snodgrass, J. G., \& Corwin, J. (1988). Pragmatics of measuring rec- ognition memory: Applications to dementia and amnesia. Journal of Experimental Psychology: General, 117, 34-50.

WAGNER, A. R. (1981). SOP: A model of automatic memory processing in animal behavior. In N. E. Spear \& R. R. Miller (Eds.), Information processing in animals: Memory mechanisms (pp. 5-47). Hillsdale, NJ: Erlbaum.

Wagner, A. R., \& Brandon, S. E. (1989). Evolution of a structured connectionist model of Pavlovian conditioning (AESOP). In S. B. Klein \& R. R. Mowrer (Eds.), Contemporary learning theories: Pavlovian conditioning and the status of traditional learning theory (pp. 149-189). Hillsdale, NJ: Erlbaum.

Wall, P. L., Botly, L. C. P., Black, C. K., \& Shettleworth, S. J. (2004). The geometric module in the rat: Independence of shape and feature learning in a food finding task. Learning \& Behavior, 32, 289-298.

White, N. M., \& McDonald, R. J. (2002). Multiple parallel memory systems in the brain of the rat. Neurobiology of Learning \& Memory, $77,125-184$.

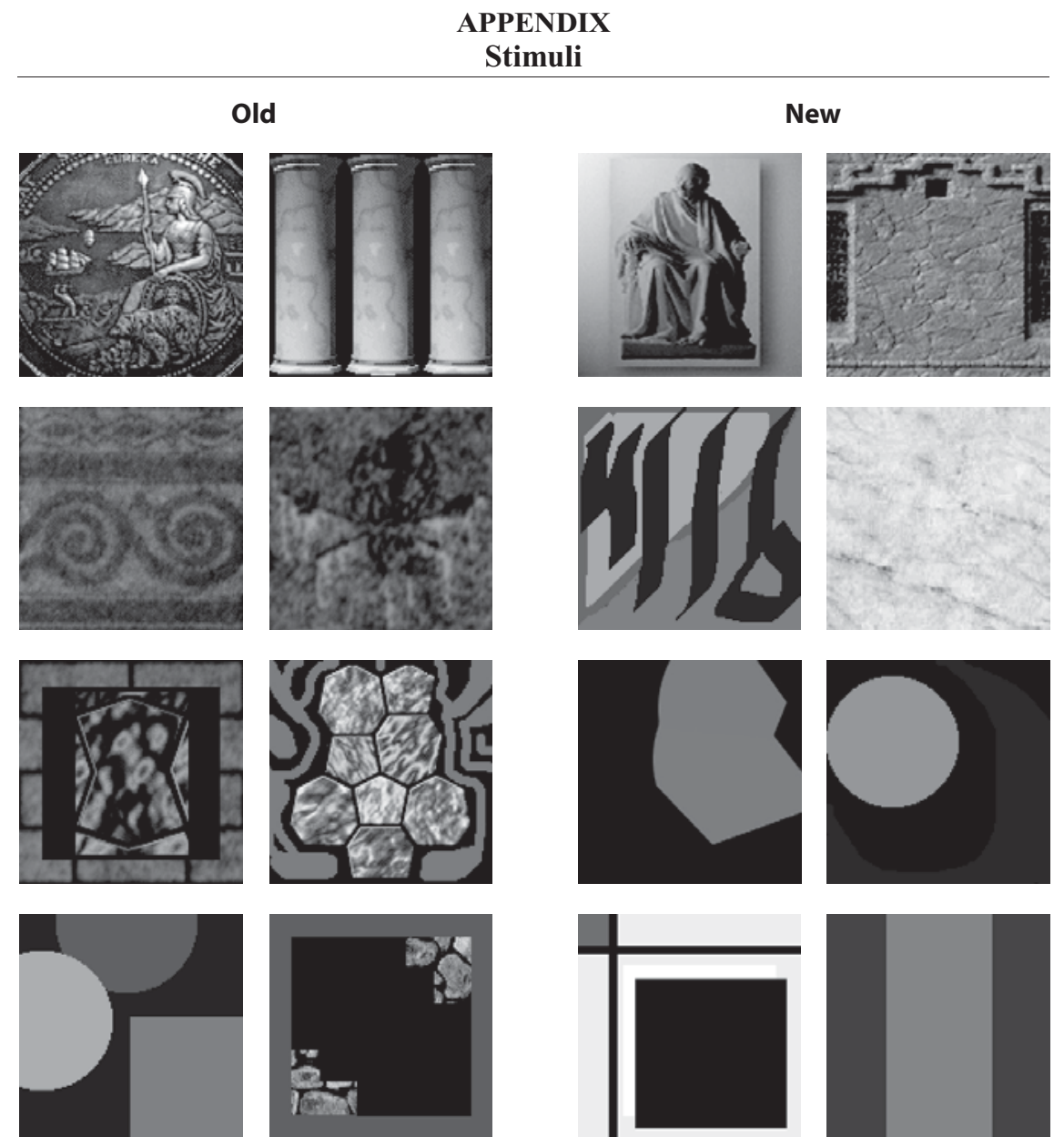

Figure A1. Stimuli for the yes/no object recognition test in Experiments 2 and 3. 


\section{APPENDIX (Continued)}

\section{Instructions for Experiments 1 and 3}

Your task is to find a large pink square, the "target". The target will be invisible until you step on it - then the target will become visible. You will know that you stepped on the target when you hear a clicking sound and you will also see the target appear on the floor - it is a pink square. You will also be trapped, so that you cannot move off the target once you are on it. The target will always be in the same location. The pictures on the wall of the room will help you to find the target. So have a good look around you each time you find the target - that will help you to quickly find the target again in the next trial.

\section{Instructions for Experiment 2}

Your task is to find a large pink square, the "target". The target will be invisible until you step on it - then the target will become visible. You will know that you stepped on the target when you hear a clicking sound and you will also see the target appear on the floor - it is a pink square. You will also be trapped, so that you cannot move off the target once you are on it.

Figure A2. Instructions used in the experiments. Only the critical part that addresses the design of the task and possible useful behavior in order to solve it is quoted here (text differences in the instructions are in italics). The remainder of the instructions provided general information - for example, how to use the keyboard for navigation and the like. This general part was identical in all the instructions used in our experiments and, thus, is not quoted here.

(Manuscript received October 9, 2007; revision accepted for publication June 23, 2008.) 